\title{
A adesão terapêutica em contexto de cuidados de saúde primários
}

\author{
John Manuel Klein ${ }^{1}$ \\ Alda da Graça André Gonçalves
}

\begin{abstract}
Resumo
A questão da adesão aos regimes de tratamento tem sido abordada, nos últimos anos, numa perspectiva biopsicossocial pelos investigadores. Nesse sentido, o presente estudo procura investigar a prevalência de determinados comportamentos de saúde, nomeadamente a adesão terapêutica e em que medida a percepção de saúde influencia este componente. Para isso, construímos e aplicámos o QIAT (Questionário Informativo de Adesão Terapêutica) aos utentes dum centro de saúde $(N=273)$. Os resultados indicam que, de um modo geral, a não-adesão não se deve ao esquecimento ou a alterações na medicação. Em relação às crenças e atitudes face aos médicos e à Medicina por parte dos utentes, existe a crença de excesso de prescrição medicamentosa, assim como uma confiança exacerbada relativa ao efeito terapêutico dos fármacos e a culpabilização dos doentes por parte dos profissionais de saúde, aquando do insucesso do tratamento.

Palavras-Chave: Adesão terapêutica; Medicação; Crenças; Centro de saúde.
\end{abstract}

\section{Therapeutical adherence in primary health care settings}

\begin{abstract}
In the last years the question of adherence to treatment regimes had been addressed by researches regarding a biopsicossocial perspective. Thus, the present study tries to analyze the prevalence of a especially component of health behaviors, namely therapeutic adherence, and at which level health perception influence that component. In order to that we developed and applied the QIAT (Informative Therapeutic Adherence Questionnaire) to a sample of Health Care Center utilizes $(N=273)$. Overall, the results showed that a negative adherence was not caused by lack of memory or changes at medication level. Further, the utilizes beliefs and attitudes regarding physicians and medicine showed the belief of an excessive medical prescription as also an exacerbated trust concerning therapeutical effects of medication by their physician and as also the physician's blaming of utilizes if treatment failure occurs.

Keywords: Therapeutic adherence; Medication; Beliefs; Health care center.
\end{abstract}

\section{Introdução}

Durante os últimos anos, vários estudos têm demonstrado que o fenómeno da adesão terapêutica se reveste da maior importância no domínio da psicologia da saúde. Efectivamente, a adesão terapêutica pode ser perspectivada como um comportamento relacionado com a saúde/doença, de tal forma que a compreensão dos factores que levam as pessoas a aderir ou não às recomendações médicas enquadra-se, assim, no âmbito da psicologia (Brannon \& Feist, 1997a; Horne, 1997), sendo a não-adesão o conceito tradicionalmente empregue para designar a falha em seguir as prescrições médicas.

Retira-se desta definição que o conceito de adesão não se circunscreve apenas a desvios no plano de tratamento, mas a comportamentos como faltar às consultas, esquecer-se de tomar a medicação prescrita, tomar medicação a mais, ingeri-la fora do tempo certo, não conhecer o nome dos fármacos a tomar, terminar o tratamento antes do prazo recomendado, entre outros aspectos; mas também com a manutenção de práticas saudáveis recomendadas pelos profissionais de saúde como comer adequadamente, fazer exercício físico suficiente, não abusar de bebidas alcoólicas, abster-se de fumar, evitar o stress (Bishop, 1994; Brannon \& Feist, 1997b; Cluss \& Epstein, 1985).

Numa revisão de vários estudos, Sheridan e Radmacher (1992) concluíram que, no geral, cerca de $50 \%$ dos pacientes não tomam os fármacos de acordo com as prescrições estipuladas, $20 \%$ a $40 \%$ não efectuam as vacinações recomendadas e $20 \%$ a $50 \%$ faltam a consultas previamente marcadas. Verificaram ainda, nos casos em que a adesão implica alterar hábitos bem estabelecidos (p. ex. deixar de fumar, diminuir ingestão de alimentos), que os índices são ainda mais altos.

De acordo com DiMatteo (1994), cerca de 38\% dos pacientes não seguiram os planos de tratamento de curta duração e mais de 45\% falharam em aderir às

${ }^{1}$ Endereço para correspondência:

Universidade do Minho/Instituto de Educação e Psicologia, Campus de Gualtar, 4750-000 - Braga - Portugal

Tel.: +351253604259 - Fax.: +351253678987

E-mail: jkein@iep.uminho.pt 
recomendações para tratamentos de longa duração. Além disso, mais de três quartos de todas as pessoas parecem não estar dispostas a aderir a estilos de vida recomendados, tais como ingerir uma dieta pobre em gorduras, evitar o tabaco e fazer exercício físico regularmente. Podemos então concluir que as pessoas falham em implementar uma vasta gama de regimes terapêuticos, desde os tradicionalmente conhecidos como curativos ou remediativos até às medidas de prevenção e redução do risco (Sarafino, 1990; Sheridan \& Radmacher, 1992).

Vários modelos ou teorias explicativas têm procurado oferecer conceptualizações capazes de explicar este comportamento e identificar os factores que determinam a tendência dos indivíduos para aderirem às recomendações médicas. Os modelos ou teorias mais frequentemente usadas no âmbito da adesão terapêutica são o modelo biomédico e várias teorias cognitivas.

O modelo biomédico foca-se, principalmente, na identificação das características dos pacientes, da doença e dos profissionais de saúde que antevêem a nãoadesão visando promover os índices de adesão terapêutica dos pacientes. Com base neste modelo, assume-se que várias características pessoais e da doença podem ser usadas para prever quem irá ou não aderir, mas não possibilita explicar as razões da não-adesão. Limita-se a identificar factores demográficos que estão relacionados com a adesão, tais como a idade, o género, o background étnico, o nível sócio-económico, e considera outras variáveis como a complexidade dos regimes de tratamento, efeitos colaterais da medicação e gravidade da doença, para determinar o grau em que cada um destes afecta a adesão (Bishop, 1994; Sarafino, 1990).

Nos modelos cognitivos, os investigadores recorreram ao conceito de auto-eficácia como o principal preditor, para explicar as diferenças encontradas a nível dos comportamentos de adesão terapêutica e para antecipar os índices de adesão numa ampla variedade de prescrições de saúde. Porém, outros autores (Miller, Wilkoff \& Hiatt, 1992) procuraram na Teoria da Acção Racional (TAR) a tentativa de prever e determinar o grau em que pacientes hipertensos recentemente diagnosticados iriam aderir às recomendações relativas à dieta, tabaco, actividade física, fármacos e evitar situações stressantes. $\mathrm{Na}$ dieta, stress e exercício físico, os comportamentos de adesão eram directamente influenciados pelas instituições que, por seu lado, eram influenciadas directamente pelas atitudes dos pacientes e pela sua motivação em aderir. No que respeita à cessação do consumo de tabaco, geralmente, a motivação em aderir estava menos relacionada com a intenção de o fazer. Isto é, algumas pessoas estavam altamente motivadas para deixar de fumar, mas não manifestavam intenção de o realizar.

Relativamente à medicação, Miller et al. (1992) constataram que o modelo não apoiava as intenções, enquanto preditores imediatos da toma correcta de medicações, apesar da motivação para aderir que é fortemente influenciada pelas crenças percebidas de outros terem uma influência directa na toma das medicações. Este estudo sugere que a TAR tem, pelo menos, algum valor na predição da adesão a recomendações médicas (Brannon \& Feist, 1997a).

De acordo com a TAR, os pacientes avaliam os benefícios e obstáculos da adesão quanto à sua probabilidade de ocorrência. Esta teoria reconhece também a importância das influências sociais nos indivíduo, nomeadamente percepções acerca das expectativas dos grupos de referência, normas subjectivas e grau de motivação do indivíduo para aderir a elas.

Finalmente, o Modelo de Crenças de Saúde (MCS) assume que a interacção entre diferentes tipos de crenças entre si influencia a adesão, bem como os comportamentos protectores/preventivos ou promotores da saúde (Brannon \& Feist, 1997a; Sheeran \& Abraham, 1995). Tendo por base o MCS, as pessoas com maior probabilidade de aderir são as que se percebem a si próprias como sendo vulneráveis a uma determinada doença e acreditam que esta tem potenciais consequências graves para a saúde ou funcionamento diário. Por outro lado, as pessoas avaliam os benefícios em relação à probabilidade de prevenção da doença, cura, controlo, ou alívio de sintomas (Conrad, 1985).

Este modelo, originalmente proposto para explicar comportamentos de saúde preventivos, foi depois expandido para explicar o papel de comportamentos de doença e adesão aos regimes terapêuticos. Assim, começou a emergir uma perspectiva alternativa que encara a não-adesão como um processo de tomada de decisão racional. A maior parte das teorias tem localizado as fontes de baixa adesão na interacção médico-paciente, no conhecimento ou crenças do paciente acerca do tratamento e também, embora em menor grau, na natureza do regime ou da doença (Conrad, 1985). Por outro lado, a maior parte dos estudos acerca da adesão tem subjacente a noção de que os pacientes deveriam aderir, essa é a sua obrigação e, quando tal não acontece, a responsabilidade é em grande parte sua. Desta forma, a adesão está estreitamente ligada ao domínio por parte da Medicina e a uma perspectiva em que os pacientes por vezes são encarados como agentes passivos no acto médico. Mas, na verdade, vários estudos sugerem que os pacientes, hoje em dia, pretendem obter mais informação e mais oportunidades de se tornarem activos na sua interacção com o profissional de saúde (Donovan \& Blake, 1992).

Stimson (1974) considera que para compreender a não-adesão é importante considerar um grupo de factores muitas vezes ignorados nos estudos e que são 
os modelos ou representações individuais de doença e as crenças dos pacientes acerca dos fármacos que influenciam o uso que fazem deles. Segundo Diefenbach e Leventhal (1992 apud Horne, 1997), as respostas de coping em relação à doença, quando consideradas como o comportamento de adesão terapêutica, são em grande medida influenciadas pelas representações que os indivíduos constroem acerca da sua doença, quanto aos cinco componentes que constituem a representação da doença (identidade, duração, causas, consequências, cura/controlo). Com base nesta perspectiva, o comportamento de não-adesão pode ser encarado como uma resposta coerente do indivíduo à falta de emparelhamento entre as sua ideias e as do médico, em relação ao seu problema e/ou tratamento (Horne, 1997). Estas afirmações vão de encontro aos aspectos anteriormente referidos relativamente aos modelos explicativos dos pacientes (Diefenbach \& Leventhal, 1996). Por outro lado, as ideias ou crenças que as pessoas desenvolvem relativamente aos fármacos em geral são susceptíveis de influenciar as suas próprias decisões acerca de quais os tratamentos apropriados para uma doença específica (Echabe, Guillen \& Ozamiz, 1992; Horne, 1997).

Segundo estas perspectivas, parece poder concluir-se que os pacientes avaliam as acções dos médicos e os fármacos prescritos, conforme o que eles próprios sabem e pensam acerca da doença e da medicação. Assim, a decisão de deixar de tomar medicamentos é um método empírico-racional que os doentes usam para testar as suas perspectivas relativamente à eficácia dos fármacos, ou seja, a opção de aderir ou não às prescrições médicas é uma forma de os doentes expressarem as suas tentativas de lidar com a doença. Por todos estes aspectos, Conrad (1985) propôs o termo auto-regulação para substituir o de adesão, pois teria um carácter mais adequado (Conrad, 1985; Donovan \& Blake, 1992; Horne, 1997). A partir desta perspectiva centrada no paciente, a adesão passa então a ser encarada como um processo de auto-regulação que permite às pessoas exercer algum controlo sobre a sua doença na medida em que se vão ajustando aos significados que os medicamentos assumem nas suas vidas e não como o acto de obedecer às instruções médicas.

Com base nestas evidências, parece fazer sentido considerar que a chave para melhorar os níveis de adesão passa pelo desenvolvimento de interacções médico-paciente mais abertas e cooperativas. Para isto é necessário que os profissionais de saúde reconheçam e não subestimem as capacidades de tomada de decisão dos seus doentes, tentem compreender as suas crenças e se disponham a colaborar com eles no desenvolvimento de terapêuticas apropriadas às suas características. É ainda preciso que os pacientes tornem mais explícitas as suas Psico-USF, v. 10, n. 2, p. 113-120, jul./ dez. 2005 necessidades e expectativas, bem como as suas crenças relativamente à doença e ao tratamento e às decisões que daí retiram. Pode-se então afirmar que o aspecto central não é a adesão em si, mas o modo como os profissionais de saúde compreendem e participam nas decisões que os pacientes tomam relativamente aos seus regimes terapêuticos.

Facilmente se constata que as consequências da baixa adesão podem ser significativas no que respeita aos cuidados de saúde, da saúde dos pacientes e, mesmo, dos resultados da investigação clínica. De acordo com Cluss e Epstein (1985), a baixa adesão pode resultar no agravamento da sintomatologia e progressão da doença, no aumento de consultas de urgência, no aumento de prescrições desnecessárias de fármacos mais potentes e/ou mesmo mais tóxicos e no fracasso do tratamento. Aquando da não-adesão por parte dos pacientes, estes ficam privados dos benefícios que supostamente iriam advir do tratamento, o que pode resultar num aumento da morbilidade, no aumento do número de consultas médicas, hospitalizações e/ou despesas desnecessárias, o que representa uma utilização ineficaz do sistema de saúde. A baixa adesão pode ainda ocasionar erros no diagnóstico e no tratamento.

Existem três factores que parecem contribuir de forma decisiva para a baixa adesão, que são: 1) as características do regime de tratamento e da doença, 2) as características do paciente e 3) as características ou natureza da relação médico-paciente. Relativamente às características do tratamento e da doença, os factores mais importantes parecem ser a sua duração e a sua complexidade, aparecendo em segundo plano os efeitos colaterais do tratamento e a gravidade da doença. Assim, os pacientes aderem mais facilmente a tratamentos relativamente simples, de curta duração e que impliquem poucas mudanças nas suas rotinas habituais (Bishop, 1994). Em relação aos efeitos colaterais, vários estudos indicam que a adesão tende a ser maior quando o tratamento tem poucos efeitos secundários desagradáveis e, sobretudo, quando é eficaz no alívio dos sintomas e tem um custo relativamente baixo (Bishop, 1994; Brannon \& Feist, 1997b; Cluss \& Epstein, 1985).

No nível das características dos pacientes, os estudos têm demonstrado pouca consistência entre a adesão e algumas características demográficas como a idade, género, estado civil e estatuto sócio-económico (Cluss \& Epstein, 1985; Conrad, 1985). Os factores que mais consistentemente têm sido referidos como bons preditores do grau de adesão dos indivíduos dizem respeito às crenças pessoais acerca da doença e da eficácia do tratamento. Segundo Kelly, Mamon e Scott (1987), as crenças e percepções pessoais de saúde são factores influentes do grau em que as pessoas tomam a medicação conforme prescrita. Desta forma, geralmente, quando as pessoas acreditam que a adesão ao tratamento 
irá produzir benefícios é mais provável que sigam as recomendações médicas. Para Diefenbach e Leventhal (1996), os modelos de senso comum que os pacientes constroem acerca das suas doenças também interferem com adesão na medida em que os ajudam a dar sentido à sua experiência e proporcionam um guia para o seu comportamento subsequente, já que as pessoas orientam todas as suas acções por forma a confirmar esses mesmos modelos. A influência das crenças também se faz sentir em um nível mais vasto se tivermos em conta as normas culturais (DiMatteo, 1994). Para além das crenças pessoais, a adesão dos pacientes à terapêutica médica é muitas vezes afectada por factores cognitivos e emocionais. Efectivamente, os estudos têm referido que os pacientes se esquecem de muitas das informações facultadas pelos médicos, sobretudo aquelas que dizem respeito às instruções e recomendações. E mais, quanto maior a quantidade de informação fornecida maior a proporção de informação esquecida, e a informação recordada pelos pacientes tenderá a ser a que foi fornecida primeiro e a que eles considerem mais importante. $\mathrm{O}$ mesmo se verifica em relação à ansiedade: quanto maior for o seu nível maior será a quantidade de informação esquecida pelos pacientes (Sarafino, 1990). Por último, o apoio social parece ser um dos factores psicossociais com maior potencial predicativo da adesão, verificando-se que as pessoas que estão isoladas de outros têm uma maior probabilidade de não aderir, enquanto as pessoas com uma rede mais vasta de relações interpessoais tendem a apresentar uma maior probabilidade de seguir as recomendações médicas, particularmente quando estas envolvam mudanças nos estilos de vida (Bishop, 1994; Sarafino, 1990).

No que respeita às características da relação médico-paciente, parecem ser factores-chave as competências de comunicação e as atitudes quanto aos pacientes. $\mathrm{Na}$ realidade, uma das principais razões pelas quais os pacientes falham em implementar os procedimentos médicos é a falha do médico em fornecer informação (Bishop, 1994). O mesmo acontece em relação à adesão, já que os estudos tendem a mostrar que os médicos sobrestimam os índices de adesão dos seus pacientes (Bishop, 1994; Sarafino, 1990; Sheridan \& Radmacher, 1992). Os problemas relacionados com a comunicação podem começar logo no momento em que o médico pede aos pacientes o relato dos seus sintomas e falha em ouvir as suas preocupações. Também no momento em que o diagnóstico é estabelecido, duas situações podem interferir com a disponibilidade do paciente para atender correctamente às instruções do médico; por um lado, se o diagnóstico for pouco grave, os pacientes podem ficar aliviados e pouco motivados para aderir (ou até ouvir) as instruções do médico; se for grave, podem ficar ansiosos e essa ansiedade interferir com a sua concentração nas recomendações médicas (Brannon \& Feist, 1997b). Subjacente a estes problemas na comunicação, podem contribuir diferenças na linguagem, nível educacional ou classe social.

Assim, este estudo procura investigar a prevalência de determinados comportamentos de saúde, nomeadamente a adesão terapêutica, e em que medida a percepção de saúde influencia este componente. Para isso, foi aplicado o QIAT aos utentes do Centro de Saúde Braga I, de forma a estudar as atitudes dos mesmos em face dos médicos e da relação entre esta dimensão e as suas crenças relativas aos fármacos em geral, bem como da percepção de comportamentos de saúde.

\section{Metodologia}

Amostra

A amostra é constituída por 273 utentes do Centro de Saúde Braga I - Carandá, com idades compreendidas entre os 9 e os 80 anos $(M=45,21 ; D P=15,94)$, sendo $86(31,5 \%)$ do sexo masculino e $187(68,5 \%)$ do sexo feminino. 69,6\% (190) dos sujeitos são casados e 21,6\% (59) são solteiros. Relativamente à profissão, verifica-se uma grande heterogeneidade, sendo as classes mais representadas as seguintes: 10,3\% (28) estudantes, 9,5\% (26) reformados, 9,2\% (25) domésticas e 8,4\% (23) professores. Também no nível das doenças que os utentes referem possuir existe uma grande heterogeneidade.

\section{Instrumentos}

O Questionário Informativo de Adesão Terapêutica (QIAT) foi desenvolvido com base nos interesses teóricos e no trabalho empírico dos autores em consonância com a informação recolhida com os profissionais de saúde e os utentes de serviços prestadores de cuidados primários de saúde, de forma a adequar-se à dinâmica de funcionamento do contexto (Klein \& Gonçalves, no prelo). Diante das necessidades e especificidade do mesmo, a sua construção teve em conta que este fosse um questionário sucinto e de fácil interpretação, de modo a permitir aos técnicos obter informação relativa a três construtos centrais da Adesão Terapêutica desses contextos.

Deste modo, as componentes deste instrumento multidimensional são: 1) adesão aos medicamentos, 2) comportamentos de saúde em face da doença e 3) crenças e atitudes diante dos médicos e da medicina.

O primeiro aspecto inclui 2 questões e pretende "medir" os níveis de adesão dos utentes deste centro de saúde à medicação, incluindo a frequência com que estes alteram as dosagens prescritas pelos médicos do centro, para as adaptarem às suas circunstâncias de vida. Assim, podemos caracterizar a não-adesão pela tendência para esquecer a ingestão da medicação e para deliberadamente 
alterar as doses recomendadas pelo médico. Esta questão é um desafio para a investigação e prática na área da saúde, pois os fármacos são por excelência o meio de intervenção médica, e constituem uma das principais fontes de despesa dos países industrializados.

A segunda dimensão deste instrumento comtempla uma questão que nos remete para as estratégias a que os doentes recorrem para minimizar os sinais e sintomas da sua doença. Este aspecto permite-nos inferir como as pessoas lidam ante um problema, neste caso específico, um problema de saúde.

A terceira dimensão, constituída por 9 questões, pretende avaliar as atitudes dos utentes em relação à eficácia da medicina e dos médicos na promoção da saúde humana, através da exploração da relação médico/ utente, do centro de saúde Braga I.

No que concerne à fidelidade, o QIAT apresenta valores aceitáveis de adesão, nomeadamente à dimensão dos medicamentos $\alpha=0,69$; à dimensão dos comportamentos de saúde quanto à doença KR20 = 0,78 e à dimensão das crenças e atitudes em relação aos médicos e à medicina $\alpha=0,86$.

É ainda de referir uma questão que foi colocada também neste questionário, com o intuito de obter informação relativa acerca da percepção de saúde/ doença dos utentes que recorrem ao centro de saúde.
Esta questão é importante, pois aquilo que as pessoas experienciam relativamente à sua saúde poderá influenciar os aspectos que já anteriormente foram descritos.

\section{Procedimento}

Os QUIATs foram entregues nas secretarias de cada módulo do Centro de Saúde Braga I - Carandá, e foi pedido aos funcionários que os distribuíssem aos utentes para que estes os preenchessem enquanto esperavam pela sua consulta.

\section{Resultados}

A nossa amostra apresenta-se bipartida no que respeita à toma de medicação actual, ou seja, metade da mesma neste momento não tem qualquer prescrição terapêutica. Relativamente à frequência com que os sujeitos se esquecem de tomar a medicação (Tabela 1), constatou-se que $43,3 \%(n=116)$ raramente o fazem e que com 33,2\% (n=89) nunca ocorre. Porém, é de ressaltar que $21,3 \%(n=57)$ dos sujeitos referem que por vezes se esquecem de tomar a sua medicação. Um outro aspecto importante da adesão à medicação prende-se ao cumprimento da prescrição médica e, aqui, constatou-se que $75,7 \%(n=196)$ dos doentes nunca alteram a sua medicação sem consultarem primeiro o seu médico.

Tabela 1 - Adesão aos medicamentos

\begin{tabular}{lccccc}
\hline & Nunca & Raramente & Às vezes & Muitas vezes & Quase sempre \\
\hline $\begin{array}{l}\text { Às vezes eu esqueço-me de tomar os } \\
\text { medicamentos }\end{array}$ & $33,2 \%$ & $43,3 \%$ & $21,3 \%$ & $1,9 \%$ & $0,4 \%$ \\
$\begin{array}{l}\text { Às vezes eu altero as doses da minha } \\
\text { medicação sem consultar o meu médico }\end{array}$ & $75,7 \%$ & $15,8 \%$ & $7,3 \%$ & $0,8 \%$ & $0,4 \%$ \\
\hline
\end{tabular}

No que concerne aos comportamentos de saúde face à doença da amostra estudada $(n=273)$ (Tabela 2), verificou-se que, ao serem questionados acerca do que fazem quando começam a sentir os primeiros sintomas de estarem doentes, $63,6 \%$ dos utentes esperam para ver se melhoram, $32,3 \%$ sujeitos tomam medicação que têm em casa e $21,9 \%$ procuram ajuda junto de conhecidos e familiares. Apenas uma minoria procura conselhos em jornais ou revistas $(3 \%)$ ou faz outras coisas $(3,4 \%)$. Mas é principalmente junto dos médicos $(78 \%)$ que os sujeitos procuram esclarecer as suas queixas, enquanto os farmacêuticos são para $33,1 \%$ dos sujeitos a pessoa a consultar em caso de doença. É ainda de ressaltar que $93,7 \%$ dos casos continuam a trabalhar.

Tabela 2 - Comportamentos de saúde em relação à doença

\begin{tabular}{lcc}
\hline & Sim & Não \\
\hline Espera para ver se melhora & $63,3 \%$ & $36,4 \%$ \\
Deixa de trabalhar para descansar mais & $6,3 \%$ & $93,7 \%$ \\
Toma medicação que tem em casa & $32,3 \%$ & $67,7 \%$ \\
Procura conselho em revistas, jornais, etc. & $3,0 \%$ & $97,0 \%$ \\
Fala com pessoas conhecidas ou familiares & $21,9 \%$ & $78,1 \%$ \\
Fala com o farmacêutico & $33,1 \%$ & $66,9 \%$ \\
Procura o médico & $78,8 \%$ & $21,2 \%$ \\
Faz outras coisas & $3,4 \%$ & $96,6 \%$ \\
\hline
\end{tabular}

Psico-USF, v. 10, n. 2, p. 113-120, jul./dez. 2005 
No que concerne às crenças e atitudes que os sujeitos apresentam em relação aos médicos e à medicina (Tabela 3), os resultados revelam que para a maioria dos sujeitos $(60,2 \%)$ os médicos não prescrevem medicamentos a mais, contrastando com 29,5\% que manifestaram uma opinião contrária. No entanto, $54,4 \%$ dos utentes acreditam que os médicos confiam demasiado nos medicamentos, ao passo que $36,3 \%$ discordam deste facto. À questão "se os médicos tivessem mais tempo com os seus doentes receitariam menos", a opinião dos sujeitos é difusa, havendo uma ligeira supremacia de sujeitos que concordam (44,3\% por oposição a $35,1 \%$ que discordam).

Tabela 3 - Crenças e atitudes em relação aos médicos e aos medicamentos

\begin{tabular}{|c|c|c|c|c|}
\hline & $\begin{array}{l}\text { Discordo } \\
\text { muito }\end{array}$ & Discordo & Concordo & $\begin{array}{c}\text { Concordo } \\
\text { muito }\end{array}$ \\
\hline Os médicos usam medicamentos a mais & $8,0 \%$ & $60,2 \%$ & $29,5 \%$ & $2,3 \%$ \\
\hline Os médicos confiam demasiado nos medicamentos & $3,1 \%$ & $36,3 \%$ & $54,4 \%$ & $6,2 \%$ \\
\hline $\begin{array}{l}\text { Se os médicos tivessem mais tempo para os } \\
\text { doentes, receitariam menos medicamentos }\end{array}$ & $9,9 \%$ & $35,1 \%$ & $44,3 \%$ & $10,7 \%$ \\
\hline $\begin{array}{l}\text { Só consulto um médico quando já não sei o que } \\
\text { fazer }\end{array}$ & $13,6 \%$ & $39,4 \%$ & $38,6 \%$ & $8,3 \%$ \\
\hline $\begin{array}{l}\text { Os médicos culpam os seus doentes se o seu } \\
\text { tratamento não resulta }\end{array}$ & $16,1 \%$ & $55,6 \%$ & $24,9 \%$ & $3,4 \%$ \\
\hline $\begin{array}{l}\text { Mesmo que se tenha de esperar muito tempo para } \\
\text { consultar um médico, vale a pena }\end{array}$ & $8,4 \%$ & $16,0 \%$ & $64,5 \%$ & $11,1 \%$ \\
\hline Os médicos sabem o que é melhor para nós & $1,9 \%$ & $13,3 \%$ & $75,4 \%$ & $9,5 \%$ \\
\hline $\begin{array}{l}\text { Se eu ficar doente, é o meu próprio comportamento } \\
\text { que determinará a rapidez com que vou ficar bom } \\
\text { de novo }\end{array}$ & $2,7 \%$ & $26,5 \%$ & $58,5 \%$ & $12,3 \%$ \\
\hline $\begin{array}{l}\text { Manter um contacto regular com o meu médico é a } \\
\text { melhor forma que eu tenho de evitar a doença }\end{array}$ & $3,4 \%$ & $15,5 \%$ & $63,0 \%$ & $18,1 \%$ \\
\hline
\end{tabular}

No que se refere à questão da urgência com que os utentes recorrem ao médico quando se sentem doentes, 38,6\% deles só recorrem à ajuda médica quando já não sabem o que fazer relativamente à sua doença; por oposição, 39,4\% dos sujeitos referem consultar logo o médico. Mais de metade dos utentes, $55,6 \%$, acham que os médicos culpam os utentes se o tratamento não funciona. No entanto, para $24,9 \%$ dos utentes este facto não ocorre. Mesmo que tenham de esperar muito para consultar um médico, $64,5 \%$ dos utentes consideram que vale a pena esperar, enquanto 16\% discordam desta situação.

A maioria dos sujeitos $(75,4 \%)$ considera que os médicos sabem o que é melhor para eles; em oposição, 13,3\% discordam desta afirmação. A maioria dos utentes, $58,5 \%$, considera que se ficarem doentes será o seu próprio comportamento que determinará a rapidez com que ficarão bons de novo, enquanto para $26,5 \%$ este aspecto não ocorre. Para a maioria dos sujeitos (63\%), a melhor forma de evitarem doenças é manter um contacto regular com o médico.

Quando inquiridos em relação ao seu estado actual de saúde, $52,4 \%$ dos utentes inquiridos consideram a sua saúde normal, $24,3 \%$ encaram a sua saúde como boa e, $15,4 \%$ referem-se a ela como sendo má.

\section{Discussão}

Tendo em conta os resultados obtidos, eles justificam e apoiam o facto de que a questão da adesão aos regimes de tratamento situa-se na interface entre o paciente, a família e o médico (Sheridan \& Radmacher, 1992) em vez de ser uma característica do paciente, da família ou do profissional de saúde.

No que concerne à não-adesão por esquecimento da toma de medicação ou por incumprimento da prescrição médica, os resultados demonstram que estes factores não explicam os comportamentos de nãoadesão por parte dos utentes deste Cento de Saúde. As ideias ou crenças que as pessoas desenvolvem acerca dos fármacos em geral, são susceptíveis de influenciar as suas próprias decisões acerca de quais os tratamentos mais apropriados para uma determinada doença (Echabe et al., 1992; Horne, 1997). Neste contexto específico, podemos então salientar que os utentes possuem informação adequada relativamente ao conhecimento das suas doenças e possíveis tratamentos, o que os leva, pelo menos numa primeira fase, a aderirem às prescrições terapêuticas dos seus médicos de família.

Relativamente às crenças e atitudes em relação aos médicos deste centro e à medicina nele praticada, os 
resultados obtidos revelam que embora os utentes pensem que os médicos não prescrevem fármacos a mais, eles confiam demasiado neles e culpam os doentes aquando do seu insucesso terapêutico. Estes factos são consistentes com a maior parte dos estudos acerca da adesão e que têm subjacente a noção de que os pacientes deveriam aderir, que é essa a sua obrigação e que, quando tal não acontece, a responsabilidade é em grande parte sua. Desta forma, a adesão está intimamente ligada à dominância da medicina e a uma perspectiva em que os pacientes tendem a ser encarados como recipientes passivos no acto médico. No entanto, várias evidências sugerem que os pacientes de hoje pretendem obter mais informação e ser mais activos na sua interacção com os profissionais de saúde (Donovan \& Blake, 1992). Daqui se infere a importância e a necessidade de uma abordagem biopsicossocial, pois os doentes são sujeitos activos no processo terapêutico e como tal necessitam de ser considerados e assumidos como parceiros em toda a interacção terapêutica, tanto na consulta médica como fora dela. Então, os comportamentos de não-adesão podem ser vistos como respostas coerentes dos indivíduos à falta de emparelhamento entre as suas ideias e as do médico relativamente aos seus problemas e/ou tratamentos (Horne, 1997).

Constatou-se que a maioria dos utentes espera para ver se melhora aquando da manifestação dos primeiros sintomas de uma doença. E, quando procura aconselhamento, ele é principalmente realizado pelo médico; embora, o aconselhamento por familiares e amigos se constitua também como um meio a ter em conta. Subjacente a isto situa-se o modo como os utentes, quando são confrontados com as perspectivas médicas e os tratamentos prescritos, iniciam, desde logo, um processo racional de análise dos custos e benefícios de cada tratamento, pesando os benefícios esperados (alívio sintomático) contra a gravidade dos sintomas e os riscos percebidos do tratamento (efeitos secundários, dependência, tempo e esforço envolvidos, estigma,...) de acordo com as crenças e teorias leigas de que já dispõem (Donovan \& Blake, 1992).

A maioria dos utentes percepciona a sua saúde como normal. Este facto poderá ser explicado com os resultados obtidos, uma vez que os sujeitos cumprem na sua grande maioria as prescrições terapêuticas e raramente se esquecem de tomar a medicação. Para mais, estes apresentam-se como participantes activos do seu processo de cura, promovendo um locus de controlo interno que promove um coping activo face à doença, pois consideram que os seus comportamentos poderão ter um papel importante na sua saúde.

Por último gostaríamos de deixar duas orientações, uma no nível da prática e outra no nível de futuras investigações. No que concerne à prática, recomendamos aos profissionais de saúde para incluírem na sua avaliação e diagnóstico a história cultural do indivíduo/ família, bem como enfatizar a importância da relação médico-doente, para que, deste modo, os problemas de não-adesão possam ser identificados desde o início da relação, pois acreditamos que é no nível interpessoal e intersistémico que as soluções para estes problemas surgirão, e daí a necessidade de se contemplar uma intervenção multidisciplinar tomando o modelo biopsicossocial como pano de fundo. Quanto a futuras investigações, recomendamos a realização de estudos longitudinais que contemplem a percepção de saúde, as crenças e comportamentos de saúde e o todo entorno sócio-cultural de cada sujeito.

\section{Agradecimentos}

Os autores agradecem aos utentes que voluntariamente participaram neste estudo, bem como à Direcção do Centro de Saúde pelos meios disponibilizados.

\section{Referências}

Bishop, G. D. (1994). Health psychology: Integrating mind and body. Boston: Allyn and Bacon.

Brannon, L. \& Feist, J. (1997a). Seeking health care. Em Health psychology: An introduction to behaviour and health (pp. 46-58). Pacific Grove, CA: Brooks \& Cole.

Brannon, L. \& Feist, J. (1997b). Adhering to medical advice. Em Health psychology: An introduction to behaviour and health (pp. 68-82). Pacific Grove, CA: Brooks \& Cole.

Cluss, P. A. \& Epstein, L. H. (1985). The measurement of medical compliance in the treatment of disease. Em Paul Karoly (Ed.). Measurement strategies in health psychology (pp. 403-433). New York: John Wiley and Sons.

Conrad, P. (1985). The meaning of medications: Another look at compliance. Social Science and Medicine, 20(1), 29-37.

Diefenbach, M. A. \& Leventhal, H. (1996). The common sense model of illness representations: Theoretical and practical considerations. Journal of Social Distress and the Homeless, 5(1), 11-38.

DiMatteo, M. R. (1994). Enhancing patient adherence to medical recommendations. Journal of the American Medical Association, 271, 79-83.

Donovan, J. L. \& Blake, D. R. (1992). Patient noncompliance: Deviance or reasoned decision-making? Social Science and Medicine, 34(5), 507-513. 
Echabe, A. E., Guillen, C. S. \& Ozamiz, J. A. (1992). Representations of health, illness and medicines: Coping strategies and health promoting behaviour. British Journal of Clinical Psychology, 31, 339-349.

Horne, R. (1997). Representations of medication and treatment: Advances in theory and treatment. Em Petrie, K. \& Weinman, J. (Eds.). Perceptions of health and illness. Current research and applications (pp. 155-189). London: Harwood Academic Publishers.

Kelly, G. R., Mamon, J. A. \& Scott, J. E. (1987). Utility of the health belief model in examining medication compliance among psychiatric outpatients. Social Science and Medicine, 25(11), 1205-1211.

Klein, J. \& Gonçalves, A. (no prelo). Propriedades psicométricas do Questionário Informativo de Adesão Terapêutica.

Miller, P., Wilkoff, R. \& Hiatt, A. (1992). Fishebein's model of reasoned action and compliance behaviour of hypertensive patients. Nursering Research, 41, 104-109.

Sarafino, E. P. (1990). Health psychology: Bio psychosocial interactions. New York: John Wiley \& Sons.

Sheeran, P. \& Abraham, C. (1995). The health belief model. Em M. Conner \& P. Norman (Eds.). Predicting health behaviour. Research and practice with social cognition models (pp. 23-61). Buckingham: Open University Press.

Sheridan, C. L. \& Radmacher, S. A. (1992). Health psychology. Challenging the biomedical model. New York: John Wiley \& Sons.

Stimson, G. V. (1974). Obeying doctor's orders: A view from the other side. Social Science and Medicine, 8, 97-104.

Recebido em abril de 2005

Reformulado em setembro de 2005 Aprovado em outubro de 2005

Sobre os autores:

John Manuel Klein é licenciado em Psicologia pela Universidade do Minho (Portugal), onde atualmente é aluno de doutoramento em Psicologia Clínica. Tem publicado e apresentado alguns trabalhos nas seguintes áreas de interesse: Psicopatologia, Processos e Resultados Terapêuticos, Avaliação Psicológica e Neurociências.

Alda da Graça André Gonçalves é licenciada em Psicologia pela Universidade do Minho (Portugal), onde atualmente está completando o Mestrado em Psicologia Clínica. Nos últimos anos, tem-se dedicado ao estudo de aspectos relacionados com as relações e adesão terapêutica, as perturbações do comportamento alimentar e as perturbações de sono. 